\title{
Kernos
}

Revue internationale et pluridisciplinaire de religion grecque antique

$24 \mid 2011$

Varia

\section{Giovanni TOSETTI, Unioni divino-umane. Un percorso storico-religioso nel mito greco arcaico}

\author{
Gabriella Pironti
}

\section{OpenEdition \\ Journals}

\section{Édition électronique}

URL : http://journals.openedition.org/kernos/1974

DOI : 10.4000/kernos. 1974

ISSN : 2034-7871

\section{Éditeur}

Centre international d'étude de la religion grecque antique

\section{Édition imprimée}

Date de publication : 1 janvier 2011

Pagination : 341-343

ISSN : 0776-3824

\section{Référence électronique}

Gabriella Pironti, « Giovanni tosetTı, Unioni divino-umane. Un percorso storico-religioso nel mito greco arcaico », Kernos [En ligne], 24 | 2011, mis en ligne le 17 octobre 2011, consulté le 21 septembre 2020. URL : http://journals.openedition.org/kernos/1974 ; DOI : https://doi.org/10.4000/kernos.1974 
Il ne m'appartient pas de juger les connaissances de C.C. en termes de neurologie et de génétique, mais force est de constater que l'aisance et la précision dont il fait preuve en ces domaines sont remarquables. De surcroit, dans la mesure où il est question avant tout de principes épistémologiques, le propos ne porte généralement pas sur des questions techniques qui seraient inaccessibles aux non initiés. D'autre part, le but de cet ouvrage, comme tout essai, est de stimuler le débat, sans viser l'exhaustivité, et c'est dans cette optique qu'il doit être lu. Le lecteur ne manquera donc pas de soulever des questions par lui-même. Par exemple, la critique globale de la subordination de la génétique au profit financier permet difficilement de se faire une idée précise des conditions de travail des scientifiques dans le domaine de la recherche universitaire. Il reste donc à voir la portée que cette réflexion aura, d'un point de vue pragmatique, sur les chercheurs en génétique eux-mêmes. De plus, le parallèle entre les apports prométhéens et la génétique est tout à fait fondé, à cette divergence près : le récit concernant Prométhée s'opérant « après coup », les techniques qu’il a transmises sont jugées indispensables à l'homme - qui ne conçoit pas, par exemple, de vivre sans feu. Le cas de la génétique pose un problème différent, puisque nous ne savons pas encore jusqu'où cette technique va nous mener. Mais sans doute est-ce justement cette différence qui est intéressante. C.C. ouvre la voie à un débat que l'avenir et les incessants progrès technologiques rendront peut-être encore plus inévitable, tout en utilisant des outils inattendus mais néanmoins pertinents : les textes grecs anciens.

Aurian Delli Pizzi

(F.R.S.-FNRS - Université de Liège)

Giovanni TOSETTI, Unioni divino-umane. Un percorso storico-religioso nel mito greco arcaico, Cosenza, Edizioni Lionello Giordano, 2008. 1 vol. 14,5 × 20,5 cm, vii+515 p. (HIER A. Collana di studi storico-religiosi, 10). ISBN : 978-88-86919-26-3.

Cet ouvrage est issu d'une thèse de Doctorat en Histoire religieuse que l'A. a soutenue à l'Université de Messina (Italie) en 2003 et représente une importante étape d'un parcours de recherche que l'A. a poursuivi aussi par la suite ${ }^{1}$. Comme Concetta Scibona Giuffré le signale dans la préface, l'A. est l'« estrema propaggine della scuola di U. Bianchi», dont l'héritage est bien présent dans la perspective scientifique adoptée dans ce livre. L'originalité de la démarche consiste, quant à elle, dans la tentative de mettre en regard cet héritage avec les interprétations $\mathrm{du}$ « mythe » et des pratiques discursives des Grecs qui ont été offertes ces dernières décennies par, et dans le sillage de, Marcel Detienne et Claude Calame. Le cadre méthodologique est précisé dans l'introduction : par l'analyse des récits d'époque archaïque qui thématisent la fin de l'âge héroïque et, avec elle, de la race des demi-dieux, l'A. se propose de vérifier l'existence, en Grèce ancienne, d'un patrimoine de récits qui pourraient à bon droit être définis comme « mythes ». Afin de démontrer la pertinence de la catégorie de « mythe », de plus en plus remise en question aussi par les hellénistes, l'A. se propose d'identifier dans les sources littéraires analysées une série de traditions irréductibles aux circonstances énonciatives particulières. Le noyau thématique stable de ces traditions véhiculerait la vision que les Grecs d'époque archaïque avaient développée d'une époque révolue, l’âge héroïque, caractérisée par une temporalité spécifique et par l'intimité des relations entre les dieux et les hommes. Le «mythe » dont l'A. suit les traces est plus particulièrement celui qui concerne à la fois la naissance des demi-dieux, descendants directs (mais aussi indirects) des unions entre immortels et mortels, et leur disparition, programmée par les dieux, à la suite des guerres de Thèbes et de Troie. Tout au long de la démonstration, le mot «mythe » est systématiquement remplacé par «le temps raconté », dont

1 Cf. G. TOSETTI, «La dernière génération héroïque : un parcours historico-religieux et sémio-narratif, d'Hésiode au ps-Apollodore », Kernos 19 (2006), p. 113-130. 
l'A. peut ainsi évaluer les interactions diverses avec «le temps de la narration » où se situent les performances des aèdes. La matière est répartie en douze chapitres, dont les premiers sont consacrés aux principaux récits qui mettent en scène les unions divino-humaines et le destin des fils nés de ces relations asymétriques. Les chapitres suivants reviennent sur des aspects ponctuels liés à ce thème : la terminologie employée, à partir du qualificatif hemitheos, ou bien le statut du héros en tant qu'objet de mémoire, dans les récits comme dans le culte. Dans les derniers chapitres, l'A. analyse les sources qui structurent en catalogues les unions divinohumaines et souligne la fonction fondatrice à la fois des demi-dieux et des récits qui en glorifient les hauts faits. Deux conclusions (dont l'une spécifiquement consacrée à confirmer la pertinence de la catégorie de «mythe »), une riche bibliographie et trois précieux index complètent cet ouvrage, qui se signale par la solide érudition que l'A. mobilise dans ses analyses et par le choix à contre-courant de retrouver le continent perdu du « mythe " grec.

Malgré la présentation quelque peu «académique », ponctuée par un apparat de notes de bas de page parfois disproportionné (la p. 166 en est un exemple flagrant) et par de nombreuses répétitions, le lecteur pourra suivre aisément l'effort argumentatif de l'A., porté par la volonté de mettre de l'ordre dans une matière narrative foisonnante. Les œuvres exceptionnelles accomplies par la génération des héros dans «le temps raconté » sont intrinsèquement liées, selon l'A., à la part divine dont ils héritent, dans la mesure où la plupart, sinon la totalité, des acteurs de cette génération se rattacherait à un ancêtre immortel. Une grande attention est réservée aux destins d'Achille et d'Énée, nés l'un comme l'autre de l'union d'une déesse immortelle et d'un homme mortel; leur généalogie est d'autant plus emblématique que ces deux héros non seulement ont été engendrés chacun par une mère divine, respectivement les déesses Thétis et Aphrodite, mais que tous deux appartiennent par leurs pères, Pélée et Anchise, à des lignages qui remontent en dernière instance à Zeus en personne. Les unions de Zeus avec des femmes mortelles constituent en effet un volet fondamental de l'enquête : à ce propos, l'A. souligne le fort impact généalogique des entreprises amoureuses du dieu souverain, montrant comment les poètes archaïques, par le recours à la structure en catalogues, ont eux-mêmes thématisé l'ensemble de ces rencontres et mis en valeur la descendance qui en est issue. Parmi les héros engendrés par Zeus, une place de choix est réservée à Héraclès, dont l'A. suit l'histoire exceptionnelle à partir des récits d'époque archaïque jusqu’à la tragédie que lui a consacrée Euripide : ses célèbres travaux sont replacés sur l'arrière-plan d'un univers encore chaotique qu'Héraclès contribue à définir et à stabiliser; la puissance de fondation, qui est reconnue par l'A. comme un trait primordial de la figure des hemitheoi et d'Héraclès plus particulièrement, aurait sa source dans la nature en partie divine qui caractérise la génération héroïque. Le statut exceptionnel de ces êtres suspendus entre l'Olympe des dieux et la terre des hommes ainsi que leur disparition légitimeraient le recours à la notion de «mythe ». Selon l'A. cette conclusion s'imposerait dans la mesure où, d'une part, le «temps raconté » mobilise une temporalité « autre », caractérisée par une relation "autrement» intime avec la sphère supra-humaine, et d'autre part, que ce temps révolu une fois pour toutes fonde le bic et nunc du « temps de la narration ».

L'A. signale à maintes reprises l'aspect problématique qui est inhérent aux unions entre immortels et mortels, telles qu'elles sont racontées par les sources d'époque archaïque : le bonheur perpétuel des dieux Olympiens se trouverait menacé par la proximité excessive avec les êtres humains, puisque les premiers souffrent à cause de la destinée mortelle des enfants issus de leur mélange avec les seconds. Les déesses, par exemple, ressentent comme une humiliation le fait de partager le lit des mortels et de leur donner des enfants, tout en révélant par la suite une sollicitude maternelle envers ces derniers : c'est le cas d'Aphrodite qui, dans le principal hymne ps.-homérique en son honneur, subit comme une punition l'union avec Anchise et l'engendrement d'Énée; c'est également le cas de Thétis poussée par les dieux contre sa volonté à devenir la femme légitime d'un mortel et la mère d'Achille. Le triste sort de Tithonos, enlevé par la déesse Éos qui obtient pour son amant l'immortalité mais pas la jeunesse éternelle, atteste 
l'incompatibilité profonde entre nature divine et nature humaine. À l'asymétrie de ces unions dans le «temps raconté » correspond en effet dans le «temps de la narration» un équilibre fondé sur la distance infranchissable entre les dieux et les hommes. Le «temps raconté » apparait donc comme téléologiquement orienté, dans la perspective de l'A., vers la disparition de la génération héroïque, suivant un axe qui conduit d'un monde où les dieux seraient encore trop proches des hommes à un monde qui se définit et s'inaugure par la séparation définitive de ces deux sphères. «Du mythe à l'histoire », aurait-on dit autrefois.

L'A. reconnait que «il limite tra l'epoca degli eventi enunciati e quella dell'enunciazione » (p. 121) est perméable, et que c'est bien dans cette époque révolue, qui est aussi une époque de «fondation », que les hommes du «temps de la narration» cherchent leurs racines et l'origine de leurs pratiques. Toutefois, dans l'effort de retrouver le «mythe » en le dégageant de la multiplicité de ses contextes d'énonciation, il finit par négliger cette perméabilité, laissant parfois dans l'ombre le dialogue qui s'instaure entre, d'un côté, les représentations véhiculées par les récits et, de l'autre, le vécu des conteurs et de leur public. Concernant Héraclès, tout en étant conscient qu'il s'agit à la fois d'un héros et d'un dieu (cf. p. 243-245), l'A. choisit d'en privilégier le statut hérö̈que pour le mettre en parallèle avec les autres hemitheoi fils de Zeus. Cela conduit à oublier la spécificité de cette figure, dont l'entrée sur l'Olympe était thématisée aussi bien dans les récits que dans l'iconographie, et cela en résonance avec les pratiques cultuelles des cités. Quoi qu'il en soit de cette réserve, cet ouvrage se distingue par la finesse déployée dans les analyses des différents témoignages littéraires et par l'attention que l'A. prête aux représentations religieuses des Grecs. Quant à la pertinence de la notion de «mythe », elle apparait moins comme le résultat d'une véritable démonstration, susceptible de réorienter cette vexata quaestio, que sous la forme de l'application systématique d'une conviction préalable.

Gabriella Pironti (Università degli Studi di Napoli 'Federico II' Centre ANHiMA [Paris])

KnoePfler Denis, La Patrie de Narcisse. Un héros mythique enraciné dans le sol et dans l'histoire d'une cité grecque, Paris, Odile Jacob, 2010. 1 vol. 14,5 × $22 \mathrm{~cm}$, 238 p. (Collège de France). ISBN : 978-2-7381-2500-2.

La relative discrétion de la figure de Narcisse dans les sources anciennes n'a d'égal que l'efflorescence de sa postérité, élégamment convoquée dans l'introduction de cet ouvrage qui se lit d'une traite, (presque) comme un roman. Il faut dire que la remarquable érudition de l'A., qui n'est jamais pesante, s'est mise au service d'une enquête dont la progression est aussi passionnante que le résultat est fragile. De quoi s'agit-il ? À l'origine de cette investigation savante se trouvent une inscription mise au jour à Érétrie, en Eubée, à l'automne 1973, une autre inscription mise au jour sur l'acropole de cette même cité en 1975, et un passage de Strabon. L'inscription de 1973 est une base chorégique malheureusement amputée de sa partie droite, dont la première ligne évoque l'identité du chorège, la troisième ligne, un joueur d'aulos, tandis que la ligne médiane porte les lettres NAPKITT..., que l'inscription de 1975, un autre monument chorégique, permet d'interpréter comme le nom Narkittis. Ce sont les premières attestations du nom de l'une des six tribus d'Érétrie, tribus qui étaient étroitement associées, comme à Athènes, à l'instauration du système démocratique vers 500. La tribu Mèkistis, seule connue avant la Narkittis, devait renvoyer à un héros local Mèkistos, et l'A. avait naguère fait l'hypothèse qu'une autre des tribus érétriennes était patronnée par le héros Orion. Trois jeunes héros auraient ainsi été choisis comme éponymes à Érétrie - on ignore qui étaient les trois autres, en dépit des efforts de l'A. pour les exhumer des sources -, alors qu'Athènes avait plutôt choisi des hommes mûrs, voire des rois. Le passage de Strabon (IX, 2, 10) est une 